\title{
Microwave Synthesis of Large Few-Layer Graphene Sheets in Aqueous Solution of Ammonia
}

\author{
Izabela Janowska ${ }^{1}(\varangle)$, Kambiz Chizari ${ }^{1}$, Ovidiu Ersen ${ }^{2}$, Spyridon Zafeiratos ${ }^{1}$, Driss Soubane ${ }^{1}$, Victor Da Costa ${ }^{2}$, \\ Virginie Speisser ${ }^{2}$, Christine Boeglin ${ }^{2}$, Matthieu Houllé ${ }^{1}$, Dominique Bégin ${ }^{1}$, Dominique Plee ${ }^{3}$, Marc-Jacques \\ Ledoux ${ }^{1}$, and Cuong Pham-Huu ${ }^{1}$ \\ ${ }^{1}$ Laboratoire des Matériaux, Surfaces et Procédés pour la Catalyse (LMSPC), UMR7515 CNRS-Université de Strasbourg 25, rue Becquerel, \\ 67087 Strasbourg Cedex 08, France \\ ${ }^{2}$ Institut de Physique et Chimie des Matériaux de Strasbourg (IPCMS), UMR7504 CNRS-Université de Strasbourg 23, rue du Loess, \\ 67037 Strasbourg Cedex 02, France \\ ${ }^{3}$ Arkema, Groupement de Recherche de Lacq (GRL), BP 34, 64170 LACQ, France \\ Received: 9 November 2009 / Revised: 10 December 2009 / Accepted: 12 December 2009 \\ (C) The Author(s) 2010. This article is published with open access at Springerlink.com
}

\begin{abstract}
Few-layer graphene (FLG) sheets with sizes exceeding several micrometers have been synthesized by exfoliation of expanded graphite in aqueous solution of ammonia under microwave irradiation, with an overall yield approaching $8 \mathrm{wt} . \%$. Transmission electron microscopy (in bright-field and dark-field modes) together with electron diffraction patterns and atomic force microscopy confirmed that this graphene material consisted mostly of mono-, bi- or few-layer graphene (less than ten layers). The high degree of surface reduction was confirmed by X-ray photoelectron and infrared spectroscopies. In addition, the high stability of the FLG in the liquid medium facilitates the deposition of the graphene material onto several substrates via low-cost solution-phase processing techniques, opening the way to subsequent applications of the material.
\end{abstract}

\section{KEYWORDS}

Graphene, transmission electron microscopy (TEM), expanded graphite exfoliation, microwaves

\section{Introduction}

Graphene has been attracting increasing scientific attention owing to its outstanding physical properties [1]. Since the first attempt to isolate an individual graphene sheet, which is a monolayer of $\mathrm{sp}^{2}$ bonded carbon [2], numerous studies have been devoted to its synthesis with the aim of improving the overall yield and reproducibility, in order to be able to exploit its various possible applications [3-5]. Graphene sheets are mostly prepared by physical methods such as micro-mechanical cleavage of graphite [2], chemical reduction of exfoliated graphite oxide [6], growth of graphene sheets on metal substrates [7], or vacuum annealing of SiC single crystals [8].

In order to improve the yield of the resulting graphene material, several different approaches have recently been proposed. Dato et al. [9] synthesized graphene sheets in a gas-phase medium using ethanol as carbon source and a microwave argon plasma reactor at atmospheric pressure. In the study of Dresselhaus and co-workers [10], large (micrometer

Address correspondence to janowskai@unistra.fr 
size) graphene sheets were synthesized by chemical vapor deposition (CVD) on a nickel film. Very recently, four new methods have been reported for unzipping a carbon nanotube lengthways [3, 11-14]. One method is based on the use of strong oxidizing reagents, e.g., $\mathrm{H}_{2} \mathrm{SO}_{4} / \mathrm{KMnO}_{4}$, whilst a second one uses the parallel insertion of metal atoms, e.g., $\mathrm{Li}$, in the presence of liquid ammonia followed by a thermal treatment. Regarding these chemical unzipping routes, problems associated with the use of hazardous chemical reagents, and thus the waste disposal, need to be addressed. A third method employs plasma etching to cut nanotubes lengthways after embedding them into a polymer film. The last method reports the synthesis of graphene by catalytic unzipping of carbon nanotubes under microwave irradiation. The as-synthesized graphene materials can be further self-assembled in a liquid medium after partial reduction leading to the formation of millimeter size superstructures similar to the ones reported by Biswas and Drzal [15].

Liquid phase exfoliation of graphite or graphite oxide (GO) by chemical solvents has also been reported by several groups. Hernandez et al. [16] reported the synthesis of graphene sheets by liquid phase exfoliation of graphite-which is the most readily available and least expensive precursor of graphene-in various solvents with yields ranging between $1 \mathrm{wt} . \%$ and $12 \mathrm{wt} . \%$. The resulting graphene sheets consisted mostly of mono- and/or few-layer graphene sheets with sizes of a few hundred nanometers. Microwave synthesis of exfoliated graphite nanoplatelets has also been reported [17]. However, the average lateral size of the graphite nanoplatelets was somewhat smaller than that obtained in the present work (lateral sizes of several micrometers).

\section{Methods}

\subsection{Materials}

The expanded graphite used in the present work was purchased from Carbone Lorraine. Ultrapure Milli- $Q^{\circledR}$ water was used for all experiments and the ammonia solution was supplied by Carlo Erba (99.9\%) and used as received.

\subsection{Graphene synthesis}

The expanded graphite (EG) with different concentrations $(0.01-1.0 \mathrm{mg} / \mathrm{mL})$ was dispersed in the liquid phase, i.e. distilled water and aqueous solution of ammonia (33 wt.\% and $16 \mathrm{wt} . \%$ ). The dispersion was homogenized by sonication in a low-power sonic bath for $30 \mathrm{~min}$. The sediment was separated by filtration and dried before further processing.

The microwave experiments were carried out on a Mars (CEM Corp.) microwave oven with the following characteristics: adjustable power between 300 and $1200 \mathrm{~W}$ and maximum temperature of $250{ }^{\circ} \mathrm{C}$. The microwave irradiation was set at $300 \mathrm{~W}$, keeping the temperature between 120 and $200{ }^{\circ} \mathrm{C}$ for $60-120 \mathrm{~min}$. The FLG sheets were formed as a suspension in the aqueous phase above the solid residual EG. In order to extract as much as possible of the FLG from the starting EG, the mixture was sonicated for $30 \mathrm{~min}$ followed by a decantation step. In the case of pure water as a solvent, the colourless suspension of the graphene sheets was separated from the starting EG by decantation or extraction with a toluene-ammonia (aq.) system, due to the low concentration of the FLG material in the solution.

\subsection{Characterisation}

The morphologies of the EG and the FLG recovered after the microwave synthesis were analyzed on a JEOL 6700 scanning electron microscope (SEM). The microstructure of the as-synthesized samples was investigated by transmission electron microscopy (TEM) on a JEOL 2100 field-emission gun (FEG) microscope operated under an acceleration voltage of $200 \mathrm{kV}$. TEM measurements were made by drop casting of the FLG dispersion onto holey carbon grids. The XPS measurements were performed on a Thermo VG spectrometer, using $\mathrm{Al} \mathrm{K} \alpha$ radiation $(h v=1486.6 \mathrm{eV})$. The base chamber pressure was $2 \times 10^{-10}$ mbar. Survey and high-resolution spectra were recorded in constant pass energy mode (100 and $20 \mathrm{eV}$, respectively). The binding energy scale of $\mathrm{SiO}_{2}$-supported graphene layers was corrected for electrostatic charging using the $\mathrm{C} 1 \mathrm{~s}$ peak at $284.5 \mathrm{eV}$ as an internal reference. A Shirley background was subtracted for all spectra. Attenuated total reflectance-Fourier transform infrared (ATR-FTIR) spectra were recorded on a Shimadzu Spectrum 100

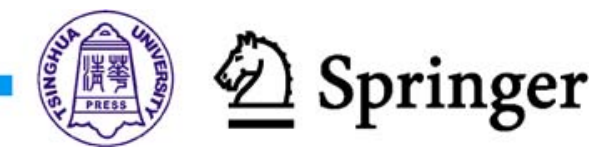


system. The AFM measurements were performed with a Digital Instruments Nanoscope. A diluted FLG dispersion in toluene $(0.1 \mathrm{mg} / \mathrm{mL})$ was spin coated (with a speed of $800 \mathrm{r} / \mathrm{min}$ ) on a $\mathrm{SiO}_{2} / \mathrm{Si}$ wafer and examined by AFM in a tapping mode. Scanning tunneling microscopy (STM) was performed in order to ascertain whether the as-synthesized FLG sheets were conductive and also to obtain more insight into the material roughness and regularity. For the thermal treatments, the sample was transferred into a separate chamber and the sample holder was connected to a tungsten filament which played the role of a heating source and allowed thermal treatments up to $600{ }^{\circ} \mathrm{C}$. The thermal treatment was carried out under a dynamic vacuum of $10^{-9}$ mbar, which provides a rapid desorption of adsorbed impurities on the FLG surface.

\section{Results and discussion}

In this work, we report the synthesis of large few-layer graphene (FLG) sheets with sizes of a few tenths to a hundred micrometers and their detailed characterization. We show that graphene material with relatively high quality, i.e., low oxygen decoration and large size, can be prepared in high yields by microwave treatment of expanded graphite in aqueous solution of ammonia at low temperatures (below $200{ }^{\circ} \mathrm{C}$ ). In a previous paper, we have reported that FLG sheets could be produced by catalytic unzipping of carbon nanotubes in water under microwave irradiation [12], although the resulting FLG possessed a relatively high amount of oxygenated functional groups on its surface. In the present work, water is replaced by ammonia which displays a higher reducibility and hence may prevent the incorporation of oxygenated groups into the synthesized FLG. Moreover, the decomposition products of ammonia are also less harmful than other chemical products used to date to synthesize graphene in liquid media. The synthesis of FLG in ammonia could also open a new field of research dealing with the production of nitrogen-doped graphene. As far as the synthesis of graphene-like materials via chemical routes is concerned, this is the first time that FLG sheets with such large sizes have been reported. Our results show that the ammonia concentration can drastically influence the exfoliation of the expanded graphite as well as the dimensions of the final FLG sheets. In addition, the FLG formed by this versatile and easily scalable method is in the form of a stable suspension in the reaction medium that can be further processed or deposited on a variety of substrates for downstream applications.

\subsection{Influence of the ammonia concentration}

The microstructure of the sample formed after microwave irradiation was investigated by transmission electron microscopy (TEM). Typical TEM images of the FLG sheets at different magnifications are presented in Fig. 1. A low-magnification TEM micrograph (Fig. 1(a)) shows part of a micrometer size FLG material that is made up of smaller individual graphene sheets. The FLG sheets constituting the assembly exhibit a relatively sharp border (Fig. 1(a)) and statistical TEM analysis indicates that their average size is about $10 \mu \mathrm{m}$ (see Section S-4 in the Electronic Supplementary Material (ESM)). General TEM views of the graphene sheet assembly show the crumpled structure of the FLG sheet (Figs. 1(b) and 1(c)). Such crumpled structure allows the sheet to accommodate the high aspect ratio of the material without scrolling $[18,19]$. The highresolution TEM micrograph taken at the border of the FLG sheet (Fig. 1(d)) clearly shows the number of graphene layers of the FLG, i.e., five layers. A quantitative analysis is possible in this case due to the particular morphology of the graphene sheets, whereas they are usually scrolled and partially folded at their edges [20]. By statistical analysis of a large number of graphene flakes, we can conclude that the graphene sheets formed by exfoliation of expanded graphite in a ammonia solution (33 wt.\%) are made up of no more than ten layers.

Furthermore, the size of the as-synthesized FLG sheets is also influenced by the ammonia concentration in the solution (see Fig. S-2 in the ESM). At high ammonia concentrations, extremely large graphene sheets with a lateral size exceeding a hundred micrometers were observed, whereas at lower ammonia concentrations, the average size of the FLG was centered around $10 \mu \mathrm{m}$. It seems that the FLG sheets retain their planar morphologies on the TEM grid after the evaporation of the solution. This suggests that the large graphene sheets can be directly deposited on 

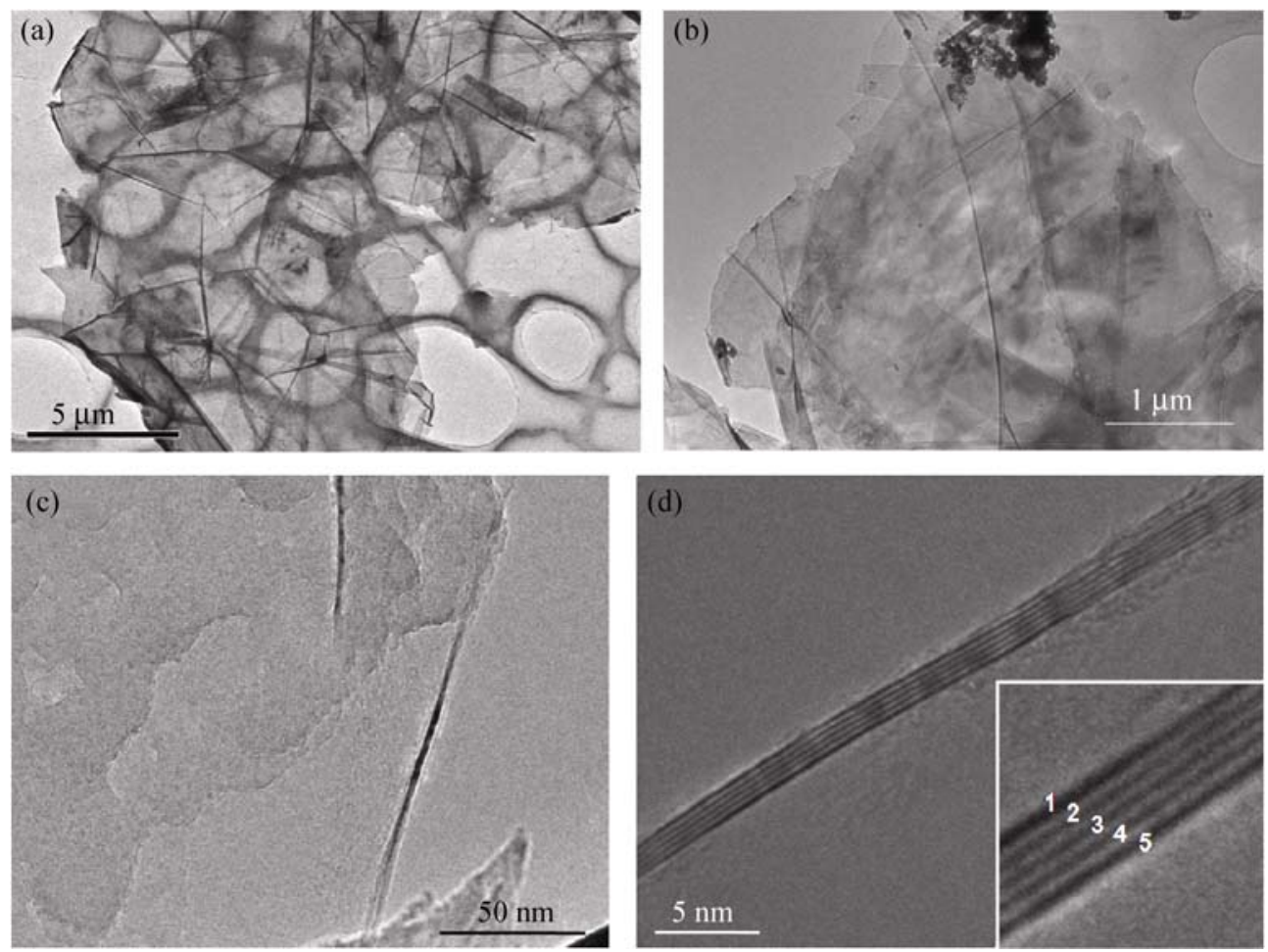

Figure 1 (a) General view of the as-synthesized graphene sheet (using an aqueous solution with 33 wt.\% of ammonia) with a width of several micrometers. (b) Closer TEM view of the FLG sheets showing that they are made up of several individual sheets with crumpled morphologies due to their high aspect ratio. (c) Border view of the graphene sheet showing the curled structure of the sheet. (d) Highmagnification TEM view of the curled border showing the number of graphene layers forming the graphene sheet. In this micrograph, there are five layers spaced with an interplanar distance of ca. $0.34 \mathrm{~nm}$

planar substrates for relevant applications. Again, typical TEM images (Fig. 2(a)) show that the edges of the sheets are generally scrolled and partially folded due to the high surface tension needed for the material to maintain its planarity over a large distance. Indeed, perfect 2-D atomic crystals cannot exist as such without scrolling, unless they are of limited size or contain many crystal defects [21]. The crystallographic structure of the sheet was determined using the selected area electron diffraction (SAED) technique which probes the whole reciprocal lattice of the sample. The SAED pattern of an assembly of three superposed sheets (Fig. 2(b)) is presented in Fig. 2(c) and corresponds to the superposition of three hexagonal lattices, one principal one with strong reflections and two others rotated slightly in-plane with respect to the first one. The typical sixfold symmetry of the pattern comes from the crystallographic structure of the graphenecomposed by carbon atoms arranged in a strictly 2-D honeycomb-in real space. To label the diffraction peaks, we use the Miller-Bravais indices $\{h k i l\}$ of graphite, putting the last index $l=0$. The two main distances determined by frequency analysis of the spots in the innermost and the next hexagon are $2.13 \AA$ (corresponding to the $\{1100\}$ spots) and $1.23 \AA$ ( $\{1120\}$ spots), respectively. Such a diffraction pattern suggests the presence of several layers in the graphene sheet assembly (see Section S-4 in the ESM). High-resolution TEM images next to the edge confirm that this FLG is made of two double layer graphene sheets, visualized as two sets of dark lines running in a parallel direction (Fig. 2(d)). Such FLG sheets could be formed by van der Waals forces during the stabilization of the material in solution. It is thought that these sheet assemblies were formed by $\pi$-stacking of the graphene material formed during the synthesis or during the cooling step. In fact, these aggregates can be easily re-dispersed by mild sonication.

In order to evaluate the typical number of graphene layers in the dispersed sheets, SAED was utilized to 

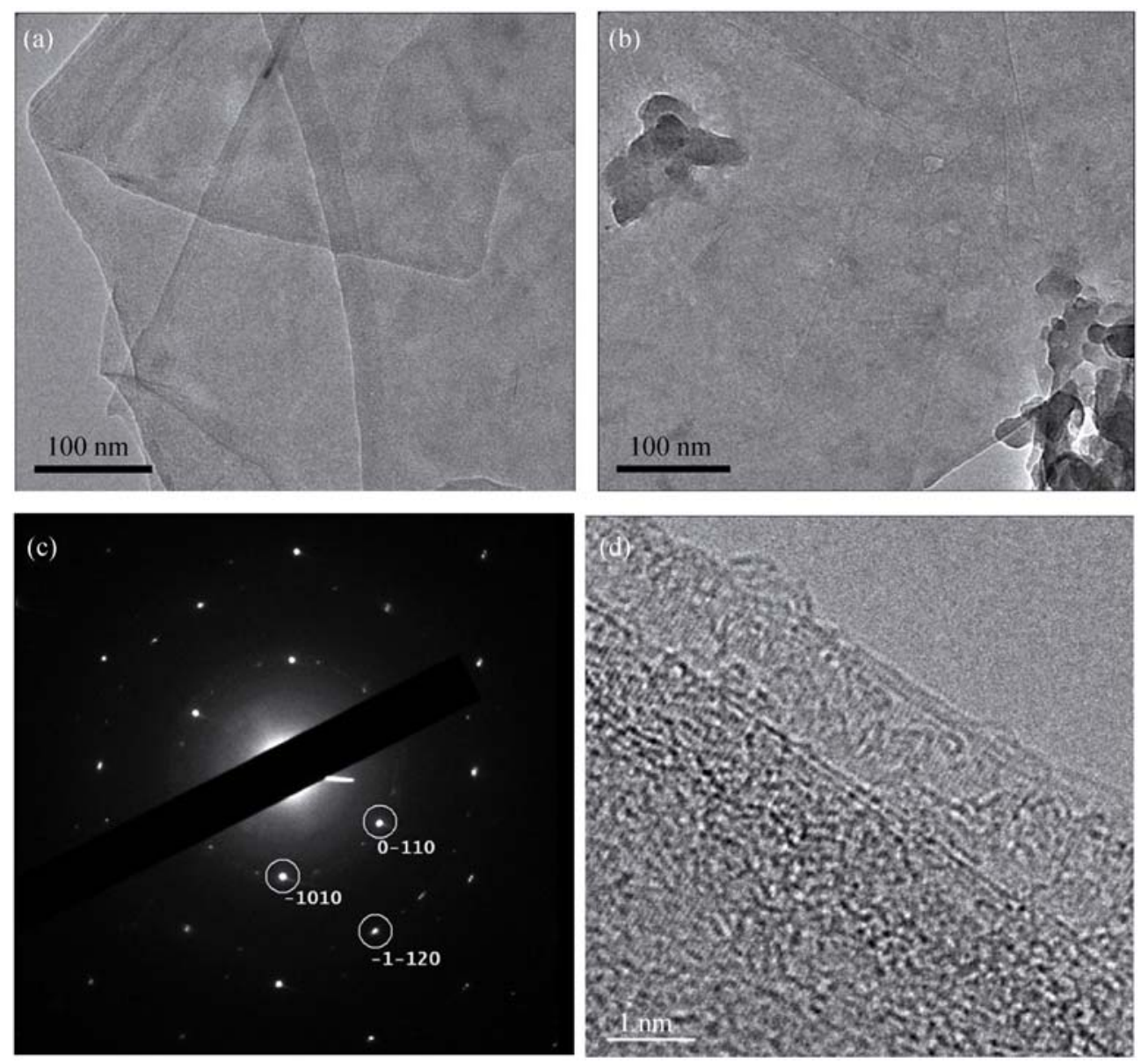

Figure 2 (a) Border view of the FLG sheets showing that each individual sheet is partly folded onto itself. (b) TEM image of an assembly of three superimposed and relatively flat graphene flakes with slight in-plane disorientations between them. (c) Selected area electron diffraction (SAED) pattern of the three graphene sheets depicted in (b). The pattern is indexed using the Miller-Bravais indices for graphite with the Bragg reflections of the innermost hexagon of (0-110) type (2.13 $\AA$ spacing) and those of the next hexagon of (-1-120) type (1.23 $\AA$ spacing). (d) High-resolution TEM micrograph of the curled border revealing the superposition of two double graphene sheets. The spacing measured between the two contrasted lines is close to $0.34 \mathrm{~nm}$

analyze several FLG sheets from different samples. This technique allows confident identification of single graphene layers by comparing the peak intensities from the innermost hexagon of the diffraction pattern with those of the next one [16]. In particular, if the multi-layers retain the Bernal $(\mathrm{AB})$ stacking of the material source, which is generally the case for few-layer sheets prepared from graphite, the $\{2110\}$ spots appear to be more intense than the $\{1100\}$ spots, whereas for single graphene layers this intensity ratio is reversed. Such an analysis was performed on typical graphene flakes, focusing on their edges. The TEM images suggest the presence of two individual sheets superposed at the edge of the flake (Fig. 3(a)). This observation is confirmed by the analysis of the diffraction pattern that shows the presence of two parallel hexagonal lattices slightly rotated around the perpendicular axis of the hexagon (Fig. 3(a) insert). By comparing the intensity of the $\{2110\}$ and $\{1100\}$ spots belonging to each hexagonal lattice, we can conclude that one of the sheets is a single-layer while the other is few-layer. Moreover, the separation in reciprocal space of the spots belonging to the two lattices allows one to visualize each graphene sheet individually, by selecting one of the two equivalent spots with the objective aperture and thus obtaining the corresponding dark field image. As a result it is possible to visualize either or both graphene sheets (by selecting both spots, Fig. 3(b)), or only one of them (Figs. 3(c) and 3(d)). 

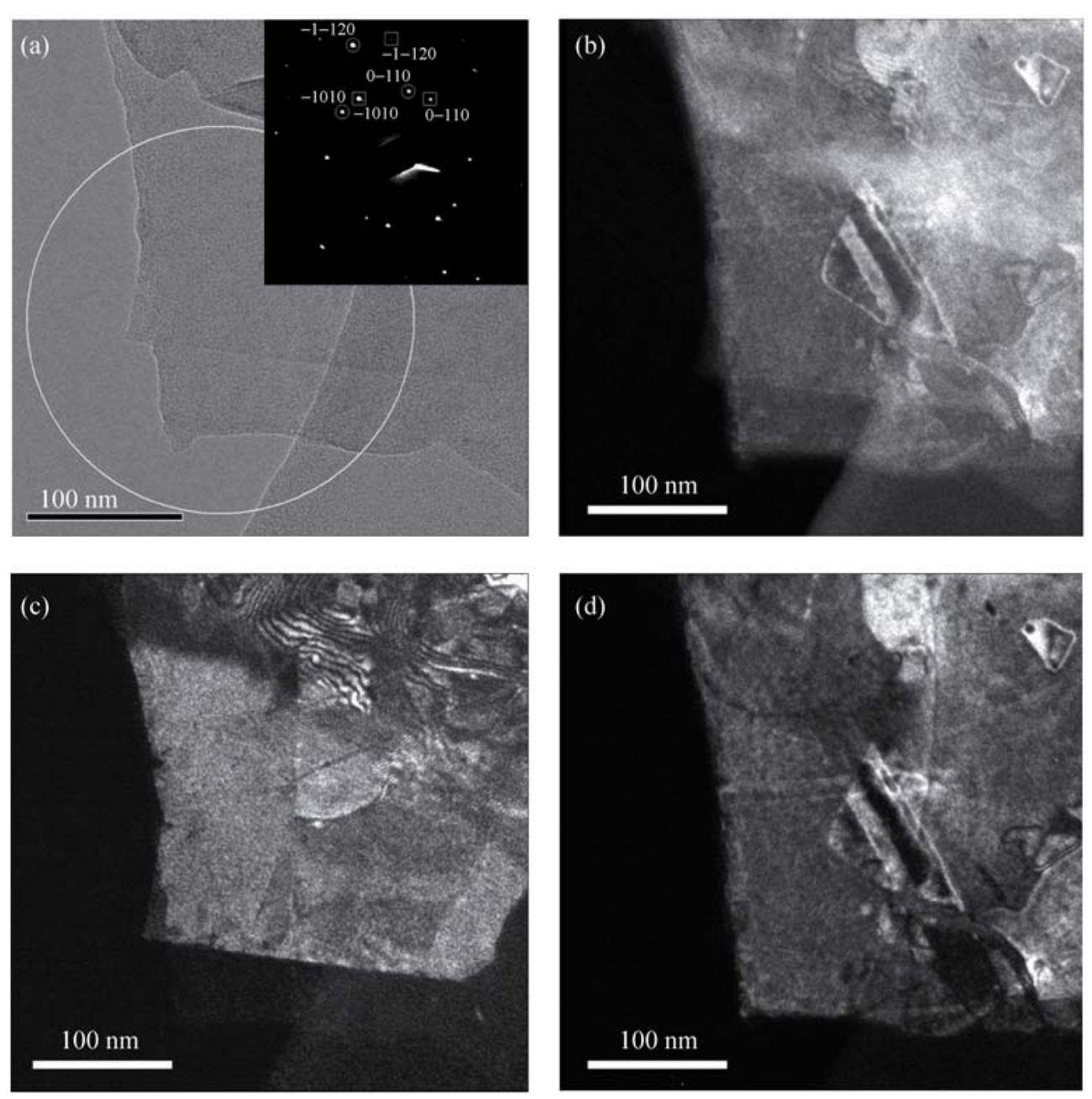

Figure 3 (a) Representative TEM image of an area where only two superposed and relatively flat graphene sheets are present. The SAED pattern taken with an aperture of $250 \mathrm{~nm}$ on the area marked by the white circle is shown in the insert. The Bragg reflections are indexed using the Bravais-Miller indices for graphite. The diffraction pattern is composed of two individual reciprocal lattices corresponding to the two superposed sheets. (b) Dark field image obtained by selecting the two (-1010) diffraction peaks: both graphene sheets are visible. (c, d) Dark fields images obtained by selecting each of the (-1010) diffraction peak separately which allows individual imaging of the two superposed sheets. These images correspond to the multilayer and the monolayer graphene sheets, respectively

Graphene sheets formed by reaction in water are thicker with smoother borders those synthesized in aqueous solution of ammonia, allowing high-resolution micrographs to be obtained without damaging the sheets with the electron beam (see Fig. S-5 in the ESM). According to the observed results one can state that the nature of the liquid medium has a substantial influence on both the morphology and the microstructure of the graphene layers. TEM analysis carried out on several samples synthesized in aqueous solution of ammonia indicated that the resulting FLG sheets were relatively homogeneous in thickness, with a maximum of ten layers. The results suggest that expanded graphite has been extensively exfoliated to give only few-layer graphene sheets with a yield of around $8 \mathrm{wt} . \%$.

The electrical conductivity of the FLG, recovered after $1 \mathrm{~h}$ of settling, and spin coated (with a speed of $800 \mathrm{r} / \mathrm{min}$ ) on a glass substrate with patterned gold was also measured. The thickness of the FLG film deposited onto a $\mathrm{SiO}_{2} / \mathrm{Si}$ wafer was ca. $400 \mathrm{~nm}$. The electrical conductivity of the sample deposited at room temperature was $2.5 \mathrm{~S} / \mathrm{m}$, which is comparable to that reported in other work, and steadily increased after reduction of the sample in flowing $\mathrm{H}_{2}$ at $200{ }^{\circ} \mathrm{C}$ for $2 \mathrm{~h}$, eventually reaching $250 \mathrm{~S} / \mathrm{m}$ [22]. Such results 
can be attributed to the removal of the oxygenated functional groups decorating the FLG edges, as suggested in the Ref. [13]. The conductivity of the as-synthesized FLG is relatively high compared to that of activated charcoal $\left(1 \times 10^{-7} \mathrm{~S} / \mathrm{m}\right)$ [23] but is still low compared to that of graphite $\left(3 \times 10^{4} \mathrm{~S} / \mathrm{m}\right)$ [24]. However, the conductivity of the FLG obtained in the present work is still high in comparison to that of the graphene obtained by solvothermal synthesis where a conductivity of only $0.067 \mathrm{~S} / \mathrm{m}$ was obtained [22].

\subsection{Proposed mechanism for the exfoliation}

Tang et al. [25] have reported a new method to synthesize graphene by rapid quenching of pristine highly oriented pyrolytic graphite (HOPG) in an aqueous solution of $\mathrm{NH}_{4} \mathrm{HCO}_{3}$. The exfoliation phenomenon was attributed to the formation of cracks on the HOPG surface during the rapid quenching followed by infiltration of $\mathrm{NH}_{4} \mathrm{HCO}_{3}$ into the material. Subsequent decomposition of $\mathrm{NH}_{4} \mathrm{HCO}_{3}$ (see Eq. (1)) leads to the formation of steam and carbon dioxide which help the exfoliation process.

$$
\mathrm{NH}_{4} \mathrm{HCO}_{3} \text { (aq.) } \longrightarrow \mathrm{NH}_{3}(\mathrm{~g})+\mathrm{CO}_{2}(\mathrm{~g})+\mathrm{H}_{2} \mathrm{O}(\mathrm{g})
$$

Based on the results of Tang et al., a mechanism can be proposed to explain the influence of the ammonia on the exfoliation of the graphite. It is expected that the high wetting of the graphite surface by the ammonia solution enhances the infiltration of the liquid between the graphene planes and thus, during the microwave irradiation, leads to an exfoliation process which produces isolated graphene sheets with different thicknesses. Indeed, the fast evaporation of water and/or the decomposition of ammonia during the microwave irradiation and the subsequent infiltration of the gaseous steam and $\mathrm{NH}_{3}$ (see Eq. (2)) within the expanded graphite matrix could give rise to an exfoliation process when the gaseous pressure overcomes the van der Waals forces between the graphene layers $(5.9 \mathrm{~kJ} / \mathrm{mol})$. Furthermore, this decomposition is thought to be almost spontaneous due to the high microwave absorption properties of the graphite, which could give rise to a fast temperature increase inside the solid matrix.

$$
\mathrm{NH}_{4} \mathrm{OH} \text { (aq.) } \longrightarrow \mathrm{NH}_{3}(\mathrm{~g})+\mathrm{H}_{2} \mathrm{O}(\mathrm{g})
$$

It is expected that at high ammonia concentrations, the liquid wetting is better and thus leads to a deeper infiltration of the ammonia solution between the graphene planes of the expanded graphite, causing the exfoliation of graphene sheets of larger sizes. Again, liquid infiltration in the interlayer galleries of the graphene planes inside the expanded graphite can be put forward to explain the observed results (see Section S-1 in the ESM).

\subsection{Surface characteristics of the few-layer graphene sheets}

The electronic structure of the graphene is strongly influenced by basal-plane or edge functionalization by oxygenated groups. In order to detect the presence of any oxygenated groups, $\mathrm{X}$-ray photoelectron spectroscopy (XPS) and attenuated total reflectance-Fourier transform infrared (ATR-FTIR) spectroscopy were performed. Characteristic C 1s and N 1s core level spectra are presented in Fig. 4 . The $C$ 1s spectrum of the graphite/graphene sample shows a narrow peak at $284.5 \mathrm{eV}$ indicating the presence of $\mathrm{sp}^{2}$ hybridized C $-\mathrm{C}$ bonds, typically assigned to graphite (Fig. 4(c)) [26]. On samples synthesized in ammonia, a low concentration of nitrogen species is detected (atomic fraction of nitrogen $\sim 1 \%-2 \%$ ) as indicated by the $\mathrm{N} 1 \mathrm{~s}$ spectra (inset of Fig. 4(d)). The binding energy of the $\mathrm{N} 1$ s peak at $400.2 \mathrm{eV}$ corresponds to nitrogen atoms bonded to $\mathrm{sp}^{2}$ carbon, and can be attributed to nitrogen atoms substituting for carbon in the graphene sheets [27]. It should be noted that the expected contribution of carbon-bonded nitrogen species in the $\mathrm{C} 1 \mathrm{~s}$ spectra is not more than $2 \%$, which is far below the uncertainty of the fitting procedure (estimated around 10\%). As expected, on samples synthesized in non-ammonia containing solutions, nitrogen is not detected in the $\mathrm{N} 1 \mathrm{~s}$ spectra.

An important factor determining the quality of graphene is the degree of oxidation, which affects the electrical properties of the material [28]. Graphene oxide involves bonding of carbon with various oxygen functional groups like hydroxyl $(-\mathrm{OH})$, keto $(\mathrm{C}=\mathrm{O})$, carboxyl $(-\mathrm{COOH})$ or epoxy groups. These species have a clear fingerprint in the $C 1$ s region, since their 


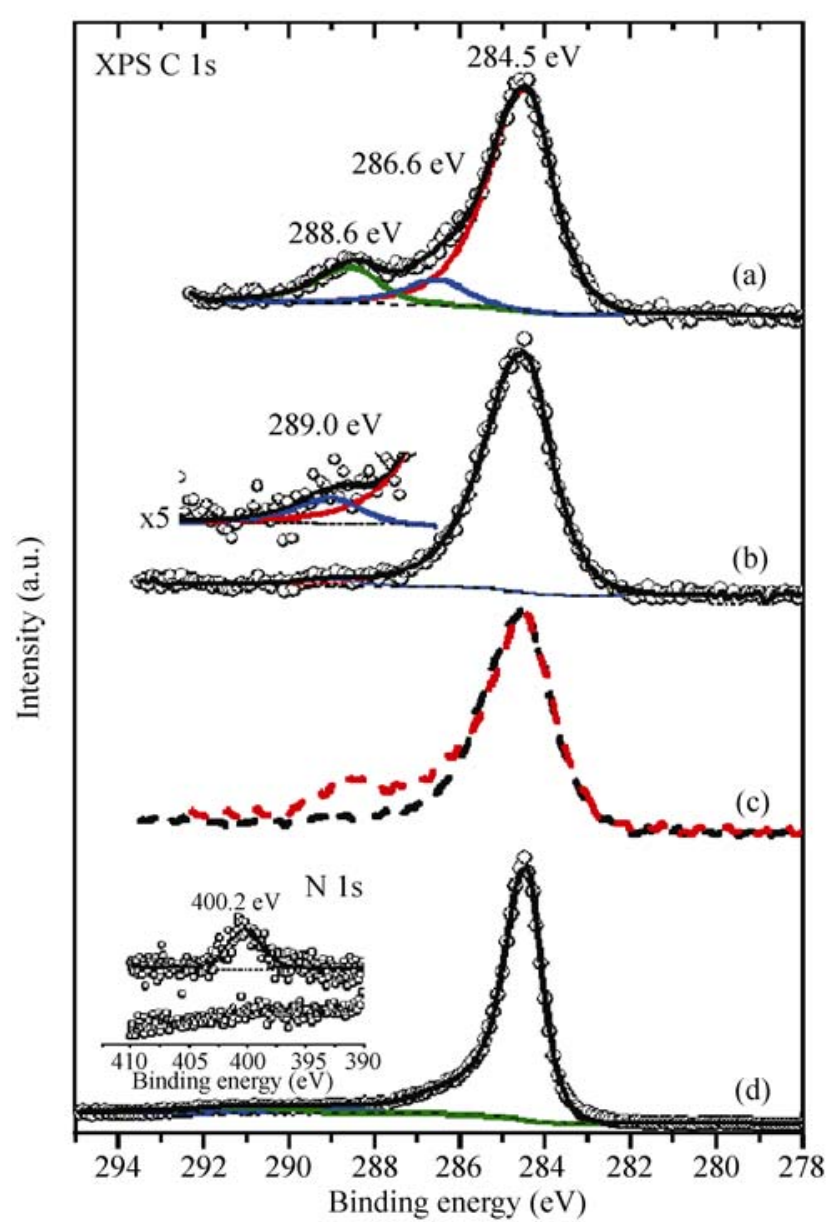

Figure 4 XPS C 1s spectra of the graphene sheets synthesized in (a) pure water and (b) $33 \mathrm{wt} . \%$ aqueous solution of ammonia. (c) Direct comparison between the two spectra ((a) and (b)) highlights the presence of a high amount of oxygenated functional groups on the FLG surface (a) synthesized without ammonia. (d) The C 1s spectrum of an unsupported graphene + graphite mixture is presented at the bottom as a reference spectrum. The FLG samples were deposited as a thin film on a $\mathrm{SiO}_{2} / \mathrm{Si}$ substrate $(1 \mathrm{~cm} \times 1 \mathrm{~cm})$ and subsequently dried at $10^{-7}$ mbar at room temperature. (d, left) Inset spectrum: the $\mathrm{N}$ 1s peaks for samples prepared in ammonia solution and pure water

binding energies are shifted between 1.5 and $4 \mathrm{eV}$ from the main graphene carbon peak. The $\mathrm{C} 1 \mathrm{~s}$ spectra of graphene layers supported on $\mathrm{SiO}_{2}$, prepared in pure water (Fig. 4(a)) and in a $33 \mathrm{wt}$.\% aqueous solution of ammonia solution (Fig. 4(b)) are presented in Fig. 4. For the sake of clarity, a direct comparison between the two XPS spectra, obtained from the FLG synthesized in water and in aqueous solution of ammonia is also presented in Fig. 4(c). The comparison confirms the higher concentration of the oxygenated functional groups on the FLG surface synthesized in water than on that synthesized in aqueous solution of ammonia. The increased width of the peaks of the $\mathrm{SiO}_{2}$ supported graphene sheets (Fig. 4(a)) compared to those of the graphene/graphite sample (Fig. 4(d)) $(1.5 \mathrm{eV}$ instead of $1 \mathrm{eV})$, is probably related to the poor electrical conductivity of the $\mathrm{SiO}_{2}$ support, resulting in an electrostatic charging of around $2 \mathrm{eV}$. As in the case of the "bulk" sample, the C 1 s peak is dominated by $\mathrm{C}-\mathrm{C}$ species at $284.5 \mathrm{eV}$. For the ammonia-treated samples, only one additional minor component at $289.0 \mathrm{eV}$ is present. The binding energy of this component can be attributed to nitrogen-bonded carbon species. This is also supported by quantitative calculations which showed that the atomic fraction of the $\mathrm{C} 1 \mathrm{~s}$ peak at $289 \mathrm{eV}$ and the $\mathrm{N} 1$ s peak is close to unity [29].

For the water-treated sample, two additional components at 286.6 and $288.6 \mathrm{eV}$ are necessary to fit the overall spectrum. These species are typically assigned to keto and carboxylic carbon species, without excluding the presence of hydroxyl groups. The important information obtained by comparing the $\mathrm{C} 1$ s peaks of the samples prepared in aqueous solution of ammonia and pure water is that graphene synthesized in ammonia solution exhibits a very low content of oxygenated species and is mostly composed of graphitic carbon. Again, this information highlights the more reduced state of the graphene sheets prepared in aqueous solution of ammonia.

This high degree of reducibility was also confirmed by attenuated total reflection infrared measurements (see Fig. S-6 in the ESM) [6, 29-31]. According to these results, an almost complete lack of peaks in the $\mathrm{COOH} / \mathrm{C}-\mathrm{OH}$ stretching region $\left(3000-3700 \mathrm{~cm}^{-1}\right)$ is found for the sample synthesized in aqueous solution of ammonia, in marked contrast to the spectrum for the sample synthesized in water.

\subsection{Atomic force microscopy analysis}

The morphology and thickness of the as-synthesized FLG were examined by atomic force microscopy (AFM). The FLG with quite small lateral size, i.e., $0.2-0.4 \mu \mathrm{m}$ was well dispersed on a $\mathrm{SiO}_{2} / \mathrm{Si}$ wafer surface (Fig. 5(a)) and lay flat on the $\mathrm{SiO}_{2} / \mathrm{Si}$ wafer substrate. The thickness of the FLG in Fig. 5(a) was measured by line profile and a value of around $4.4 \mathrm{~nm}$ 
was obtained. According to Ref. [32], which indicates that the thickness of a single graphene layer is around $1 \mathrm{~nm}$, the FLG obtained in the present work contain between two and five graphene layers. FLG with larger lateral sizes, i.e., $0.6 \mu \mathrm{m}$, was also observed, as highlighted in Fig. 5(b). Statistical analysis revealed that the FLG has an average thickness ranging between 3 and $5 \mathrm{~nm}$, which corresponds to FLG containing three to five layers of graphene. It is worth mentioning that FLG with even larger lateral sizes $(>1 \mu \mathrm{m})$, as observed by TEM, was not found by AFM. This difference can be explained by the instability of such large lateral sizes of FLG on the hydrophilic $\mathrm{SiO}_{2} / \mathrm{Si}$ wafer surface. FLG with large lateral sizes have a tendency to scroll during the drying step and thus the analysis of these structures by AFM was not successful. It is expected that FLG with such large lateral sizes is easier to observe by TEM due to the presence of the underlying carbon membrane on the TEM grid which prevents them scrolling during the drying step. This hypothesis is confirmed by using a membrane-free TEM grid (see Figs. S-3(a) and S-3(b) in the ESM) in which case the large FLG readily scrolled, due to its high difficulty in maintaining a high aspect ratio 2-D structure. We are currently investigating the LangmuirBlodgett method as a way to prepare samples with better stability on the $\mathrm{SiO}_{2} / \mathrm{Si}$ wafer surface for subsequent AFM analysis.

\subsection{Scanning tunneling microscopy analysis}

The topology roughness and the electrical conductivity of the FLG sheets synthesized in aqueous solution of ammonia were measured by scanning tunneling microscopy (STM). The graphene sample was annealed in vacuum at $150{ }^{\circ} \mathrm{C}$ for $1 \mathrm{~h}$ before analysis. Topographic STM images (Fig. 5(c)) show the relatively high roughness of the sheet with a height variation of up to $5 \mathrm{~nm}$. After further annealing at a higher temperature, $400{ }^{\circ} \mathrm{C}$, the roughness was significantly decreased from 5 to around $2 \mathrm{~nm}$ (Fig. 5(d)). Such a decrease can be attributed to the removal of adsorbed species. The morphology of graphene films is dominated by elongated strips with periodic stacking which are characterized by $2 \mathrm{~nm}$ high protuberances. Meyer et al. [20] have reported that graphene sheets are stabilized by an out-of-plane deformation which can reach $1 \mathrm{~nm}$ in height, similar to the STM results in the present work. Such a corrugation becomes smaller for bilayer graphene and disappears for thicker graphene sheets.

In order to further explore the conductivity of these graphene sheets, localized current vs. bias potential curves were acquired (Fig. 5(e)). The curve acquired from $-3 \mathrm{~V}$ to $+3 \mathrm{~V}$ clearly exhibits an ohmic behavior over the whole potential range of conductivity $(-2 \mathrm{~V}$ to $+1.8 \mathrm{~V}$ ). The conductivity is observed at positive and negative bias, clearly indicating the ambipolarity of graphene, where carriers can be tuned between holes and electrons by supplying the requisite bias. Moreover, the signal acquired during the measurement was quite stable (low noise level) indicating that the surface probed by the tip was rather clean and lacking significant amounts of adsorbed impurities. Outside these limits, the conductivity reached saturation levels, demonstrating that all the electronic states of the FLG sheets have been probed [33, 34]. Figure 5(f) highlights the positive effect of the annealing on the conductivity: a ten-fold increase of the conductivity slope $(\mathrm{d} I / \mathrm{d} V)$ was observed when the sample was heated from $150{ }^{\circ} \mathrm{C}$ to $400{ }^{\circ} \mathrm{C}$. These results are in line with the observations already made by Kosynkin et al. [13], who also reported an increase in the conductivity of graphene nanoribbons annealed at $300{ }^{\circ} \mathrm{C}$ under $\mathrm{H}_{2} / \mathrm{Ar}$, and is also consistent with previously reported data from Hernandez et al. [16], where the authors also found a large increase in conductivity after thermal annealing of the sample. This shows that most of the conductivity comes only from the intrinsic properties of the graphene sheets and not from adsorbed impurities.

Even if it is very difficult to make assumptions concerning the tip shape and size, one can estimate a tunneling effect area of about $1 \mathrm{~nm}^{2}$ over the sample. Considering this, it is possible to make a simple calculation, assuming an ohmic conductivity along one dimension:

$$
\sigma=\frac{i_{\text {setpoint }}}{E_{\text {bias }}}\left(\frac{t}{A}\right)
$$

where $\sigma$ is the conductivity, $i_{\text {setpoint }}$ is the probe current, $E_{\text {bias }}$ is the applied bias potential, $A$ is the tunneling 


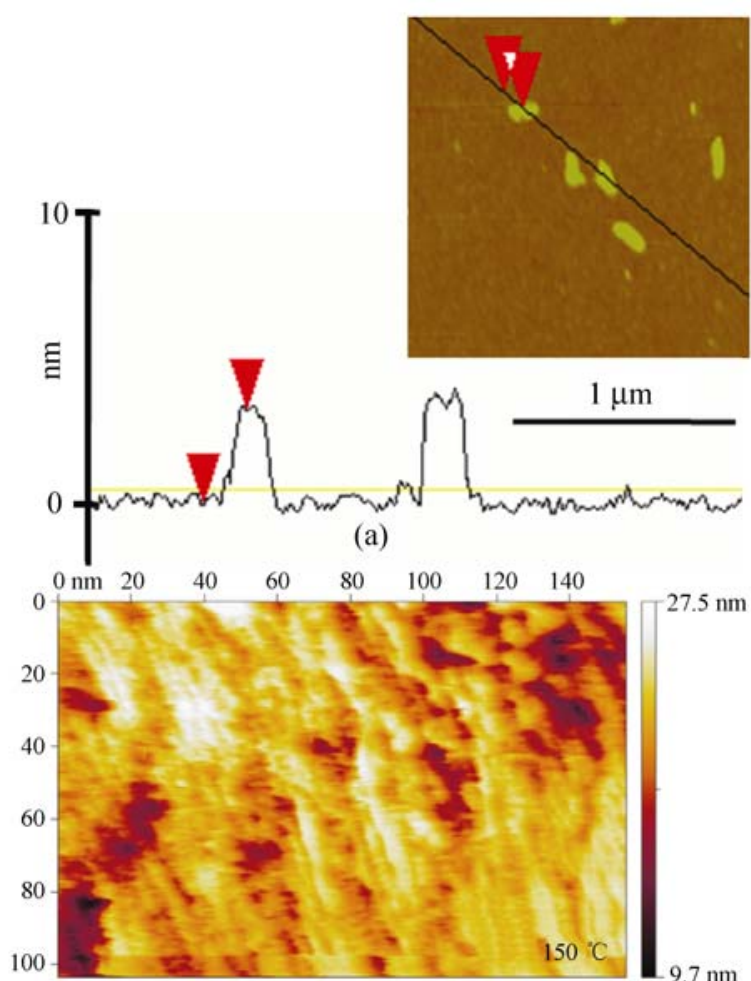

(c)

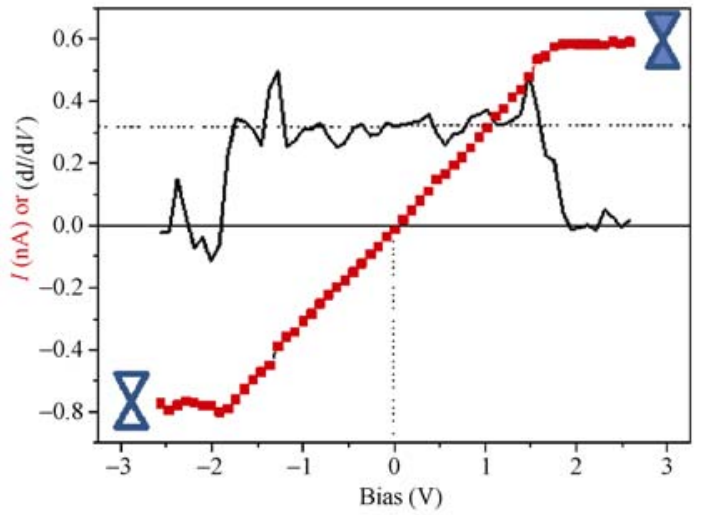

(e)

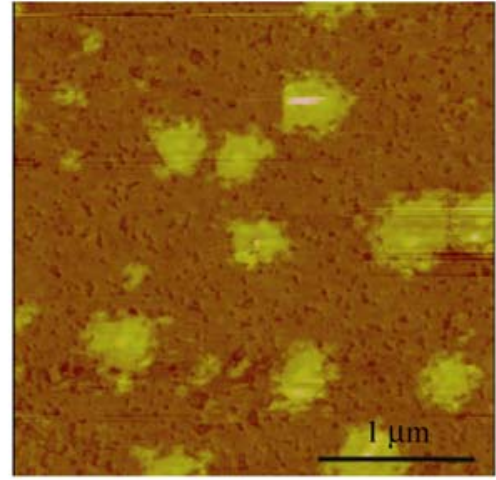

(b)

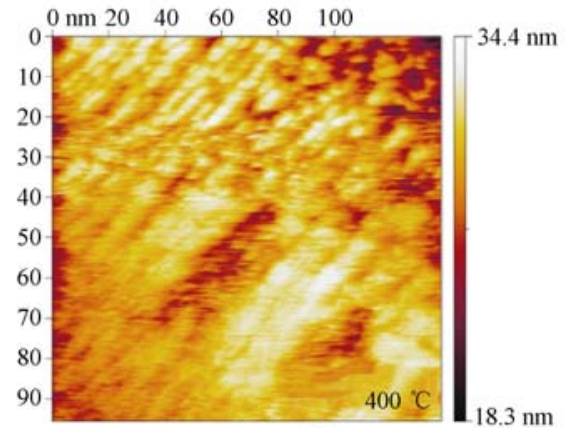

(d)

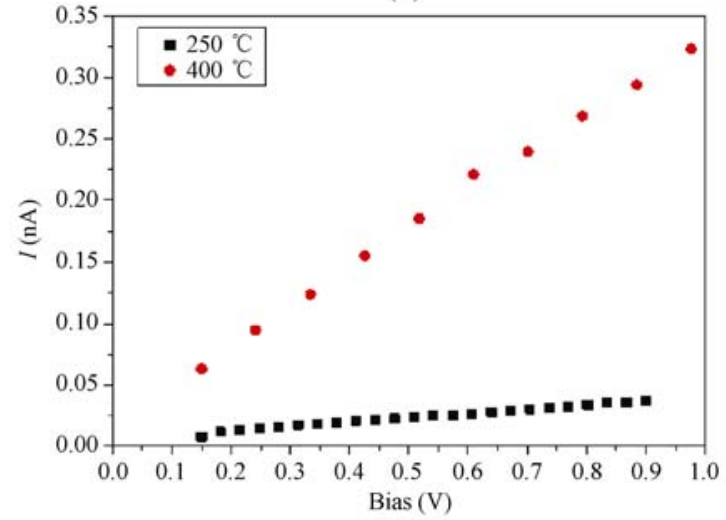

(f)

Figure 5 (a) The line scan of a small FLG illustrates the homogeneous thickness of the samples, ca. $4.4 \mathrm{~nm}$. (b) AFM images in tapping mode on a $3 \mu \mathrm{m} \times 3 \mu \mathrm{m}$ area showing an FLG with larger lateral size. (c) and (d) STM topographic images of the graphene film deposited on a Ta sample holder. The experimental conditions were $V_{\text {bias }}=-0.4 \mathrm{~V}$ and $I_{\text {tunnel }}=0.2 \mathrm{nA}$. The roughness of the FLG significantly decreases as a function of increasing annealing temperature and duration: $R=8 \mathrm{~nm}$ after annealing at $150{ }^{\circ} \mathrm{C}$ for $1 \mathrm{~h}$ and $R=4 \mathrm{~nm}$ after annealing at $400{ }^{\circ} \mathrm{C}$ for $1 \mathrm{~h}$. (e) and (f) $I / V$ curves obtained by performing the measurement at one local point and averaging over the STM image showing an ohmic behavior of the graphene film. The ambipolarity of charge transfer is demonstrated, as the conductivity of graphene is observed under negative and positive bias

effect area, and $t$ is the thickness of the sample. In spite of the obvious simplicity of this model, an estimation of the conductivity of the graphene sheets perpendicular to the graphene plane can still be calculated. This simple equation gives a conductivity of about $1000 \mathrm{~S} / \mathrm{m}$ for the sample annealed at $400{ }^{\circ} \mathrm{C}$.

\section{Conclusions}

For the first time, large size $(0.1 \mathrm{~mm})$ few-layer graphene sheets have been synthesized in aqueous solution of ammonia under microwave irradiation at relatively low temperature by exfoliation of 
expanded graphite. An exfoliation mechanism involving the following steps is suggested: (i) infiltration of the ammonia solution between the graphene sheets of the expanded graphite, and (ii) spontaneous decomposition of the ammonia solution $\left(\mathrm{NH}_{4} \mathrm{OH}\right)$ by microwave radiation to form gaseous $\mathrm{NH}_{3}$ and $\mathrm{H}_{2} \mathrm{O}$ which carry out the exfoliation. The exfoliation efficiency of this process is proportional to the concentration of ammonia in the liquid medium leading to larger graphene flakes in the case of high ammonia concentrations. A combination of TEM and electron diffraction statistical analyses performed on several regions and samples indicated that the exfoliation product consisted mostly of mono-, bi-, and few-layer $(<10)$ graphene sheets with relatively straight edges. XPS and ATR-FTIR spectra revealed the reduced state of the graphene. TEM and STM indicated that the graphene surface was not planar, but rather that some corrugations of nanometer dimensions were present. Such corrugations allow the stabilization of the whole 2-D crystal. Additional experiments using other liquid media are needed in order to fully understand the exfoliation mechanism of the expanded graphite via a spontaneous liquid-to-vapor transformation.

\section{Acknowledgements}

The TEM experiments were carried out at the Institut de Physique et Chimie des Matériaux de Strasbourg (IPCMS) facilities. Dr. Thierry Romero is gratefully acknowledged for performing the SEM experiments. Dr. Anne Boos is gratefully acknowledged for help during the microwave irradiation experiments. Cacao J. P. is gratefully acknowledged for help and stimulation during the work.

Electronic Supplementary Material: Details of the wetting behaviour of the expanded graphite by ammonia solution, the morphology of the expanded graphite, the morphological stability of the graphene sheet, a selected area electron diffraction study of agraphene monolayers, the influence of the liquid nature on the graphene morphology and thickness, and ATR-FTIR spectra are available in the online version of this article at http://dx.doi.org/10.1007/s12274-0101017-1 and accessible free of charge.

\section{References}

[1] Geim, A. K.; Novoselov, K. S. The rise of graphene. Nat. Mater. 2007, 6, 183-191.

[2] Novoselov, K. S.; Novoselov, K. S.; Geim, A. K.; Morozov, S. V.; Jiang, D.; Zhang, Y.; Dubonos, S. V.; Grigorieva, I. V.; Firsov, A. A. Electric field effect in atomically thin carbon films. Science 2004, 306, 666-669.

[3] Jiao, L. Y.; Zhang, L.; Wang, X. R.; Diankov, G.; Dai, H. J. Narrow graphene nanoribbons from carbon nanotubes. Nature 2009, 458, 877-880.

[4] Li, D.; Muller, M. B.; Gilje, S.; Kaner, R. B.; Wallace, G. G. Processable aqueous dispersions of graphene nanosheets. Nat. Nanotechnol. 2008, 3, 101-105.

[5] Ponomarenko, L. A.; Schedin, F.; Katsnelson, M. I.; Yang, R.; Hill, E. W.; Novoselov, K. S.; Geim, A. K. Chaotic Dirac billiard in graphene quantum dots. Science 2008, 320, 356-358.

[6] Stankovich, S.; Dikin, D. A.; Piner, R. D.; Kohlhaas, K. A.; Kleinhammes, A.; Jia, Y.; Wu, Y.; Nguyen, S. T.; Ruoff, R. S. Synthesis of graphene-based nanosheets via chemical reduction of exfoliated graphite oxide. Carbon 2007, 45, 1558-1565.

[7] Kim, K. S.; Zhao, Y.; Jang, H.; Lee, S. Y.; Kim, J. M.; Kim, K. S.; Ahn, J. H.; Kim, P.; Choi, J. Y.; Hong, B. H. Largescale pattern growth of graphene films for stretchable transparent electrodes. Nature 2009, 457, 706-710.

[8] Berger, C.; Song, Z. M.; Li, X. B.; Wu, X. S.; Brown, N.; Naud, C.; Mayou, D.; Li, T. B.; Hass, J.; Marchenkov, A. N.; Conrad, E. H.; First, P. N.; de Heer, W. A. Electronic confinement and coherence in patterned epitaxial graphene. Science 2006, 312, 1191-1196.

[9] Dato, A.; Radmilovic, V.; Lee, Z. H.; Phillips, J.; Frenklach, M. Substrate-free gas-phase synthesis of graphene sheets. Nano Lett. 2008, 8, 2012-2016.

[10] Reina, A.; Jia, X. T.; Ho, J.; Nezich, D.; Son, H. B.; Bulovic, V.; Dresselhaus, M. S.; Kong, J. Large area, few-layer graphene films on arbitrary substrates by chemical vapor deposition. Nano Lett. 2009, 9, 30-35.

[11] Janowska, I.; Ersen, O.; Jacob, T.; Vennégues, P.; Bégin, D.; Ledoux, M. J.; Pham-Huu, C. Catalytic unzipping of carbon nanotubes to few-layer graphene under microwave irradiation. Appl. Catal. A 2009, 371, 22-30.

[12] Janowska, I.; Pham-Huu, C.; Ersen, O.; Begin, D.; Ledoux, M. J. French Patent, 09-00100, 2009.

[13] Kosynkin, D. V.; Higginbotham, A. L.; Sinitskii, A.; Lomeda, J. R.; Dimiev, A.; Price, B. K.; Tour, J. M. Longitudinal unzipping of carbon nanotubes to form graphene nanoribbons. Nature 2009, 458, 872-876. 
[14] Cano-Marquez, A. G.; Rodriguez-Macias, F. J.; CamposDelgado, J.; Espinosa-Gonzalez, C. G.; Tristan-Lopez, F.; Ramirez-Gonzalez, D.; Cullen, D. A.; Smith, D. J.; Terrones, M.; Vega-Cantu, Y. I. Ex-MWNTs: Graphene sheets and ribbons produced by lithium intercalation and exfoliation of carbon nanotubes. Nano Lett. 2009, 9, 1527-1533.

[15] Biswas, S.; Drzal, L. T. A novel approach to create a highly ordered monolayer film of graphene nanosheets at the liquid-liquid interface. Nano Lett. 2008, 9, 167-172.

[16] Hernandez, Y.; Nicolosi, V.; Lotya, M.; Blighe, F. M.; Sun, Z. Y.; De, S.; McGovern, I. T.; Holland, B.; Byrne, M.; Gun'ko, Y. K.; Boland, J. J.; Niraj, P.; Duesberg, G.; Krishnamurthy, S.; Goodhue, R.; Hutchison, J.; Scardaci, V.; Ferrari, A. C.; Coleman, J. N. High-yield production of graphene by liquid-phase exfoliation of graphite. Nat. Nanotechnol. 2008, 3, 563-568.

[17] Drzal, L. T.; Fukushima, H. Composite material for batteries, electrodes, comprises expanded graphite obtained by heating acid intercalated graphite precursor with first polymer, dispersed in second polymer. U. S. Patent, 20060148966-A1, Feb. 27, 2006.

[18] Le Doussal, P.; Radzihovsky, L. Self-consistent theory of polymerized membranes. Phys. Rev. Lett. 1992, 69, 1209-1212.

[19] Nelson, D. R.; Peliti, L. Fluctuations in membranes with crystalline and hexatic order. J. Phys. France 1987, 48, 1085-1092.

[20] Meyer, J. C.; Geim, A. K.; Katsnelson, M. I.; Novoselov, K. S.; Booth, T. J.; Roth, S. The structure of suspended graphene sheets. Nature 2007, 446, 60-63.

[21] Mermin, N. D. Crystalline order in two dimensions. Phys. Rev. 1968, 176, 250-254.

[22] Choucair, M.; Thordarson, P.; Stride, J. A. Gram-scale production of graphene based on solvothermal synthesis and sonication. Nat. Nanotechnol. 2009, 4, 30-33.

[23] Coutinho, A. R.; Rocha, J. D.; Luengo, C. A. Preparing and characterizing biocarbon electrodes. Fuel Process. Technol. 2000, 67, 93-102.

[24] Deprez, N.; McLachlan, D. S. The analysis of the electrical conductivity of graphite conductivity of graphite powders during compaction. J. Phys. D: Appl. Phys. 1988, 21, 101-107.

[25] Tang, Y. B.; Lee, C. S.; Chen, Z. H.; Yuan, G. D.; Kang, Z. H.; Luo, L. B.; Song, H. S.; Liu, Y.; He, Z. B.; Zhang, W. J.;
Elello, I.; Lee, S. T. High-quality graphenes via a facile quenching method for field-effect transistors. Nano Lett. 2009, 9, 1374-1377.

[26] Yang, D.; Velamakanni, A.; Bozoklu, G.; Park, S.; Stoller, M.; Piner, R. D.; Stankovich, S.; Jung, I.; Field, D. A.; Ventrice, C. A.; Ruoff, R. S. Chemical analysis of graphene oxide films after heat and chemical treatments by X-ray photoelectron and micro-Raman spectroscopy. Carbon 2009, 47, 145-152.

[27] Bongiorno, G.; Blomqvist, M.; Piseri, P.; Milani, P.; Lenardi, C.; Ducati, C.; Caruso T.; Rudolf, P.; Wachtmeister, S.; Csillag, S.; Coronel, E. Nanostructured $\mathrm{CN}_{x}(0<x<0.2)$ films grown by supersonic cluster beam deposition. Carbon 2005, 43, 1460-1469.

[28] Lotya, M.; Hernandez, Y.; King, P. J.; Smith, R. J.; Nicolosi, V.; Karlsson, L. S.; Blighe, F. M.; De, S.; Wang, Z. M.; McGovern, I. T.; Duesberg, G. S.; Coleman, J. N. Liquid phase production of graphene by exfoliation of graphite in surfactant/water solutions. J. Am. Chem. Soc. 2009, 131, 3611-3620.

[29] Wei, D.; Liu, Y. Q.; Wang, Y.; Zhang, H. L.; Huang, L. P.; $\mathrm{Yu}, \mathrm{G}$. Synthesis of $\mathrm{N}$-doped graphene by chemical vapor deposition and its electrical properties. Nano Lett. 2009, 9, 1752-1758.

[30] Becerril, H. A.; Mao, J.; Liu, Z.; Stoltenberg, R. M.; Bao, Z.; Chen, Y. S. Evaluation of solution-processed reduced graphene oxide films as transparent conductors. ACS Nano 2008, 2, 463-470.

[31] Eda, G.; Fanchini, G.; Chhowalla, M. Large-area ultrathin films of reduced graphene oxide as a transparent and flexible electronic material. Nat. Nanotechnol. 2008, 3, 270-274.

[32] Li, X.; Zhang, G.; Bai, X.; Sun, X.; Wang, X.; Wang, E.; Dai, H. Highly conducting graphene sheets and LangmuirBlodgett films. Nat. Nanotechnol. 2008, 3, 538-542.

[33] Kaiser, A. B.; Gomez-Navarro, C.; Sundaram, R. S.; Burghard, M.; Kern, K. Electrical conduction mechanism in chemically derived graphene monolayers. Nano Lett. 2009, 9, 1787-1792.

[34] Lherbier, A.; Blase, X.; Niquet, Y. M.; Triozon, F.; Roche, S. Charge transport in chemically doped 2D graphene. Phys. Rev. Lett. 2008, 101, 036808. 